\title{
Esophageal Varices Hemorrhage, CTCAE 5.0
}

National Cancer Institute

\section{Source}

National Cancer Institute. Esophageal Varices Hemorrhage, CT CAE 5.0. NCI Thesaurus.

Code C146710.

A disorder characterized by bleeding from esophageal varices. 